Probability Surveys

Vol. 19 (2022) 113-128

ISSN: $1549-5787$

https://doi.org/10.1214/22-PS5

\title{
Floating bodies and approximation of convex bodies by polytopes*
}

\author{
Elisabeth M. Werner \\ Department of Mathematics, Case Western Reserve University, \\ Cleveland, Ohio, USA \\ e-mail: elisabeth.werner@case.edu
}

\begin{abstract}
We describe some results on approximation of convex bodies by polytopes. Best and random approximations are considered and compared. The geometric quantities related to the convex body, that appear naturally in such approximation questions are the affine surface areas. Those, and their relation to floating bodies, will be discussed as well.

In this survey we give only a very selective collection of results on approximation by polytopes.
\end{abstract}

MSC2020 subject classifications: 52A20, 52A40, 60B.

Keywords and phrases: Floating bodies, affine surface area, random polytopes.

Received October 2021.

\section{Contents}

1 Introduction . . . . . . . . . . . . . . . . . . . . . . . . . . 114

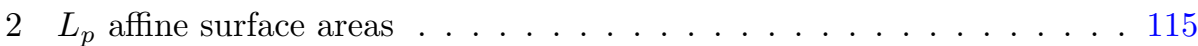

2.1 Definitions, examples and properties . . . . . . . . . . . 115

2.2 Rényi divergenes of cone measures . . . . . . . . . . . . . . . 117

3 (Weighted) floating body and surface body . . . . . . . . . . . 118

3.1 Definitions and properties . . . . . . . . . . . . . . 118

3.2 Surface body and $L_{p}$ affine surface area . . . . . . . . . . 119

4 Random polytopes of points chosen from a convex body . . . . . . . . 121

4.1 The main theorem . . . . . . . . . . . . . . . . . 121

4.2 An analysis of the main theorem . . . . . . . . . . . . . 124

4.2.1 Comparison: Best approximation and random approximation . . . . . . . . . . . . . . . . 124

$4.2 .2 \quad$ Other measures $\mathbb{P}_{f} \ldots \ldots \ldots \ldots$. . . . . . . . . . . . . . 124

4.2.3 Random polytopes and $L_{p}$ affine surface areas $\ldots \ldots .125$

Acknowledgments . . . . . . . . . . . . . . 126

References . . . . . . . . . . . . . . . . . 126

*Partially supported by NSF grants DMS-1811146 and DMS-2103482 and by a Simons Fellowship 


\section{Introduction}

How well can a convex body be approximated by a polytope? This is a central question in the theory of convex bodies, not only because it is a natural question and interesting in itself but also because it is relevant in many applications, for instance in computer vision, tomography, geometric algorithms, local theory of Banach spaces, stochastic geometry and many more. We only quote $[4,9,10$, $11,13,15,17,18,19,26,30]$.

It often involves side conditions like a prescribed number of vertices, or, more generally, $k$-dimensional faces and a requirement that the body contains the polytope or vice versa. Various metrics are used to measure accuracy of approximation. Ideally, one then wants the optimal dependence on all the parameters involved: the dimension $n$, the convex body $K$, the number of prescribed vertices or facets or $k$-faces. An example of such a result of asymptotic nature is the following remarkable theorem due to Gruber [13] in dimension $n$ and due to McClure and Vitale [24] in dimension 2 which says that for a convex body $K$ in $\mathbb{R}^{n}$ with sufficiently smooth boundary

$\inf \left\{d_{s}\left(K, P_{N}\right) \mid P_{N}\right.$ is a polytope, contained in $K$, having at most $N$ vertices $\}$

$$
\begin{gathered}
\text { is asymptotically equal as } N \rightarrow \infty \text { to } \\
\frac{1}{2} \operatorname{del}_{n-1}\left(\int_{\partial K} \kappa(x)^{\frac{1}{n+1}} d \mu_{K}(x)\right)^{\frac{n+1}{n-1}}\left(\frac{1}{N}\right)^{\frac{2}{n-1}} .
\end{gathered}
$$

Here $d_{s}$ is the symmetric difference metric (see below), $\partial K$ denotes the boundary of the convex body $K, \mu_{K}$ is the usual surface area measure on $\partial K$ and $\kappa$ is the Gauss curvature. This result tells us that when we approximate by an inscribed polytope with a fixed number of vertices $N$ the optimal dependence on $N$ in this case is $N^{-2 /(n-1)}$. The optimal dependence on the dimension is hidden in the constant $\operatorname{del}_{n-1}$. Only later it was determined that $\operatorname{del}_{n-1}$ is of the order $\sqrt{n},[12,22,23]$. The dependence on the body $K$ comes in via the affine surface area $\int_{\partial K} \kappa(x)^{\frac{1}{n+1}} d \mu_{K}(x)$. This quantity, in a sense, "measures" the boundary behavior of a convex body, so it is natural that it should appear in questions of approximation of convex bodies by polytopes. In Section 2 we describe that quantity and its generalizations in more detail.

It is only in rare special cases that a best approximating polytope can be explicitly singled out. Consequently, a common practice is to randomize: choose $N$ points at random in the convex body with respect to a probability measure $\mathbb{P}$. The convex hull of these randomly chosen points is called a random polytope.

As is demonstrated in Section 4, choosing the points randomly inside the convex body does not yield optimal dependence with respect to the number of chosen points. It is more economical to choose the points directly from the boundary of the body.

In Section 4 we quote a result by Schütt and Werner [35], which, in a nutshell, can be phrased as "random approximation is as good as best approximation". More precisely, let $\mathbb{E}(K, N)$ be the expected volume of a random polytope, the 
convex hull of $N$ randomly chosen points on $\partial K$. It is proved in [35] that there is a constant $c_{n}$ such that

$$
\begin{gathered}
\operatorname{vol}_{n}(K)-\mathbb{E}(K, N) \text { is asymptotically equal as } N \rightarrow \infty \text { to } \\
\frac{c_{n}}{2}\left(\int_{\partial K} \kappa(x)^{\frac{1}{n+1}} d \mu_{K}(x)\right)^{\frac{n+1}{n-1}}\left(\frac{1}{N}\right)^{\frac{2}{n-1}} .
\end{gathered}
$$

We will comment in Section 4 on the constant $c_{n}$ and its relation to $\operatorname{del}_{n-1}$. Here, and elsewhere, $\operatorname{vol}_{n}(K)$ denotes the volume of $K$. We observe that again affine surface area appears. Note also that affine surface area is a priori only defined for sufficiently smooth bodies so that the Gauss curvature exits. This is a drawback as we want such approximation results for all convex bodies.

It is exactly here that floating bodies, and their variants, surface bodies and weighted floating bodies come in. These bodies can be used to define affine surface area and $L_{p}$ affine surface area for all convex bodies. This will be explained in Section 3.

In this survey we give only a very selective collection of results on approximation by polytopes. For complementary reading we suggest the paper [27].

\section{2. $L_{p}$ affine surface areas}

\subsection{Definitions, examples and properties}

Throughout this note we will assume without loss of generality that the center of gravity or centroid

$$
g(K)=\frac{1}{\operatorname{vol}_{n}(K)} \int_{K} x d x
$$

of a convex body $K$ in $\mathbb{R}^{n}$ is at the origin. For real $p \neq-n$, the $L_{p}$ affine surface area $a s_{p}(K)$ of $K$ was introduced for $p>1$ in a seminal paper [21] by Lutwak and extended to all $p \neq-n$ in [36],

$$
a s_{p}(K)=\int_{\partial K} \frac{\kappa(x)^{\frac{p}{n+p}}}{\langle x, N(x)\rangle^{\frac{n(p-1)}{n+p}}} d \mu_{K}(x)
$$

and

$$
a s_{ \pm \infty}(K)=\int_{\partial K} \frac{\kappa(x)}{\left\langle x, N_{K}(x)\right\rangle^{n}} d \mu_{K}(x),
$$

where $\langle\cdot, \cdot\rangle$ is the standard inner product on $\mathbb{R}^{n}$ which induces the Euclidian norm $\|\cdot\|$ and $N(x)$ is the outer unit normal vector at $x \in \partial K$. In particular, for $p=0$ we get

$$
a s_{0}(K)=\int_{\partial K}\langle x, N(x)\rangle d \mu_{K}(x)=n \operatorname{vol}_{n}(K) .
$$

The case $p=1$

$$
a s_{1}(K)=\int_{\partial K} \kappa(x)^{\frac{1}{n+1}} d \mu_{K}(x)
$$


is the classical affine surface area which goes back to Blaschke [5]. A definition for $p=-n$ was proposed in [25].

If the boundary of $K$ is sufficiently smooth then (3) and (4) can be written as integrals over the boundary $\partial B_{2}^{n}=S^{n-1}$ of the Euclidean unit ball $B_{2}^{n}$ in $\mathbb{R}^{n}$

$$
a s_{p}(K)=\int_{S^{n-1}} \frac{f_{K}(u)^{\frac{n}{n+p}}}{h_{K}(u)^{\frac{n(p-1)}{n+p}}} d \sigma(u) .
$$

Here, $\sigma$ is the usual surface area measure on $S^{n-1}, h_{K}(u)=\max _{x \in K}\langle x, u\rangle$ is the support function of direction $u \in S^{n-1}$, and $f_{K}(u)$ is the curvature function, i.e. the reciprocal of the Gaussian curvature $\kappa(x)$ at this point $x \in \partial K$ that has $u$ as outer normal. In particular, for $p= \pm \infty$,

$$
a s_{ \pm \infty}(K)=\int_{S^{n-1}} \frac{d \sigma(u)}{h_{K}(u)^{n}}=n \operatorname{vol}_{n}\left(K^{\circ}\right)
$$

where $K^{\circ}=\left\{y \in \mathbb{R}^{n},\langle x, y\rangle \leq 1, \forall x \in K\right\}$ is the polar body of $K$. As the Gauss

curvature of a polytope is 0 almost everywhere, the $L_{p}$ affine surface area of a polytope is 0 or $\infty$, depending on $p$. Other examples are listed next. The details can be found in [36].

Example 1. Let $1<r<\infty$ and $B_{r}^{n}=\left\{\left.x \in \mathbb{R}^{n}\left|\sum_{i=1}^{n}\right| x_{i}\right|^{r} \leq 1\right\}$. Then we have

(i) For $1<r<2$ and $-\frac{n}{r-1} \leq p<-n$ and for $2<r<\infty$ and $-n<p \leq$ $-\frac{n}{r-1}$

$$
a s_{p}\left(B_{r}^{n}\right)=\infty .
$$

(ii) For all other cases with $p \neq-n, \pm \infty$ we have

$$
a s_{p}\left(B_{r}^{n}\right)=\frac{2^{n}(r-1)^{\frac{p(n-1)}{n+p}}}{r^{n-1}} \frac{\left(\Gamma\left(\frac{n+r p-p}{r(n+p)}\right)\right)^{n}}{\Gamma\left(\frac{n(n+r p-p)}{r(n+p)}\right)} .
$$

In particular, for the Euclidean unit ball $B_{2}^{n}$, for all $p \neq-n$,

$$
a s_{p}\left(B_{2}^{n}\right)=\frac{2 \pi^{\frac{n}{2}}}{\Gamma\left(\frac{n}{2}\right)}=\operatorname{vol}_{n-1}\left(\partial B_{2}^{n}\right) .
$$

The next theorem collects some of the properties of $L_{p}$ affine surface area. These properties make affine surface area a useful instrument in questions where information on the boundary of a convex body is needed. In particular, the affine isoperimetric inequalities, which are stronger than their Euclidean counterparts, are an efficient tool to detect ellipsoids.

Theorem 2. Let $K$ be a convex body in $\mathbb{R}^{n}$.

(i) For all $p \neq-n$ and for all invertible linear transformations $T: \mathbb{R}^{n} \rightarrow \mathbb{R}^{n}$

$$
a s_{p}(T(K))=|\operatorname{det}(T)|^{\frac{n-p}{n+p}} a s_{p}(K) .
$$


(ii) For $0 \leq p \leq \infty, L_{p}$ affine surface area is an upper semicontinuous functional and for $-n<p \leq 0$ a lower semicontiuous functional with respect to the Hausdorff metric.

(iii) For $p \geq 0$,

$$
\frac{a s_{p}(K)}{a s_{p}\left(B_{2}^{n}\right)} \leq\left(\frac{v o l_{n}(K)}{\operatorname{vol}_{n}\left(B_{2}^{n}\right)}\right)^{\frac{n-p}{n+p}}
$$

and for $-n<p \leq 0$,

$$
\frac{a s_{p}(K)}{a s_{p}\left(B_{2}^{n}\right)} \geq\left(\frac{\operatorname{vol}_{n}(K)}{\operatorname{vol}_{n}\left(B_{2}^{n}\right)}\right)^{\frac{n-p}{n+p}} .
$$

Equality holds in both inequalities iff $K$ is an ellipsoid. Equality holds trivially in both inequalities if $p=0$.

Property (i) was shown in [21,36]. The semicontinuity is due to Lutwak [21]. The $L_{p}$ affine isoperimetric inequalities were proved by Lutwak [21] for $p>1$ and for all other $p$ by Werner and Ye [39]. The case $p=1$ is the classical case. For $-\infty \leq p<-n$ there is an inequality as well and it was proved in [39].

\subsection{Rényi divergenes of cone measures}

Let $(X, \mu)$ be a measure space and let $d P=p d \mu$ and $d Q=q d \mu$ be probability measures on $X$ that are absolutely continuous with respect to the measure $\mu$. Then the Rényi divergence of order $\alpha$, introduced by Rényi [29], is defined for $\alpha \neq 1$ as

$$
D_{\alpha}(P \| Q)=\frac{1}{\alpha-1} \log \int_{X} p^{\alpha} q^{1-\alpha} d \mu .
$$

The integrals

$$
\int_{X} p^{\alpha} q^{1-\alpha} d \mu
$$

are also called Hellinger integrals, see e.g. [16] for those integrals and additional information.

Usually in the literature, the measures are probability measures. Therefore we normalize the measures. An important special case of Rényi divergence is the case when $\alpha \rightarrow 1$, which leads to the Kullback-Leibler divergence or relative entropy from $P$ to $Q$ (see [8]),

$$
D_{K L}(P \| Q)=D_{1}(P \| Q)=\lim _{\alpha \uparrow 1} D_{\alpha}(P \| Q)=\int_{X} p \log \frac{p}{q} d \mu .
$$

In [38] Rényi divergence was introduced for convex bodies $K$ in $\mathbb{R}^{n}$, as follows. Let

$$
p_{K}(x)=\frac{\kappa(x)}{\langle x, N(x)\rangle^{n} n \operatorname{vol}_{n}\left(K^{\circ}\right)}, \quad q_{K}(x)=\frac{\langle x, N(x)\rangle}{n \operatorname{vol}_{n}(K)} .
$$


Then

$$
P_{K}=p_{K} \mu_{K} \quad \text { and } \quad Q_{K}=q_{K} \mu_{K}
$$

are probability measures on $\partial K$ that are absolutely continuous with respect to $\mu_{K}$. These measures can be viewed as the cone measures of the convex bodies $K$ and $K^{\circ}$, respectively. We refer to [38] for the details (see also Section 4.2.2).

We then can define the Rényi divergence of $K$ of order $\alpha$ for all $\alpha$.

Definition 3. [38] Let $K$ be a convex body in $\mathbb{R}^{n}$ and let $\alpha \in \mathbb{R}, \alpha \neq 1$. Then the Rényi divergences of order $\alpha$ of $K$ are

$$
\begin{aligned}
D_{\alpha}\left(Q_{K} \| P_{K}\right) & =\frac{1}{\alpha-1} \log \left(\frac{\int_{\partial K} \frac{\kappa_{K}^{1-\alpha} d \mu_{K}}{\langle x, N(x)\rangle^{n-\alpha(n+1)}}}{n \operatorname{vol}_{n}(K)^{\alpha} \operatorname{vol}_{n}\left(K^{\circ}\right)^{1-\alpha}}\right) \\
D_{\alpha}\left(P_{K} \| Q_{K}\right) & =\frac{1}{\alpha-1} \log \left(\frac{\int_{\partial K} \frac{\kappa_{K}^{\alpha} d \mu_{K}}{\langle x, N(x)\rangle^{\alpha(n+1)-1}}}{n \operatorname{vol}_{n}(K)^{1-\alpha} \operatorname{vol}_{n}\left(K^{\circ}\right)^{\alpha}}\right) .
\end{aligned}
$$

The $L_{p}$ affine surface areas are a central part of the $L_{p}$-Brunn Minkowski theory, an extension of the classical Brunn Minkowski theory, see, e.g., [31]. A remarkable fact which connects $L_{p}$-Brunn Minkowski theory and information theory was observed in [38], namely:

$L_{p}$ affine surface areas of a convex body are exponentials of Rényi divergences of the cone measures of $K$ and $K^{\circ}$.

Theorem 4. [38] Let $K$ be a convex body in $\mathbb{R}^{n}$. Let $-\infty \leq p \leq \infty, p \neq-n$. Then

$$
\begin{aligned}
\frac{a s_{p}(K)}{n \operatorname{vol}_{n}(K)^{\frac{n}{n+p}} \operatorname{vol}_{n}\left(K^{\circ}\right)^{\frac{p}{n+p}}} & =\operatorname{Exp}\left(-\frac{n}{n+p} D_{\frac{p}{n+p}}\left(P_{K} \| Q_{K}\right)\right) \\
& =\operatorname{Exp}\left(-\frac{p}{n+p} D_{\frac{n}{n+p}}\left(Q_{K} \| P_{K}\right)\right)
\end{aligned}
$$

In particular,

$$
\begin{aligned}
\frac{a s_{1}(K)}{n \operatorname{vol}_{n}(K)^{\frac{n}{n+1}} \operatorname{vol}_{n}\left(K^{\circ}\right)^{\frac{1}{n+1}}} & =\operatorname{Exp}\left(-\frac{n}{n+1} D_{\frac{1}{n+1}}\left(P_{K} \| Q_{K}\right)\right) \\
& =\operatorname{Exp}\left(-\frac{1}{n+1} D_{\frac{n}{n+1}}\left(Q_{K} \| P_{K}\right)\right)
\end{aligned}
$$

\section{3. (Weighted) floating body and surface body}

\subsection{Definitions and properties}

For a convex body $K \subseteq \mathbb{R}^{n}$ and $0 \leq \delta<\frac{\operatorname{vol}_{n}(K)}{2}$, the floating body $K_{\delta}$ was introduced independently by Barany and Larman [3] and by Schütt and Werner 
[34], as the intersection of all halfspaces $H^{+}$whose defining hyperplanes $H$ cut off a set of volume $\delta$ from $K$,

$$
K_{\delta}=\bigcap_{\operatorname{vol}_{n}\left(H^{-} \cap K\right) \leq \delta} H^{+} .
$$

It is obvious that $K_{\delta} \subseteq K$, that $K_{\delta}$ is convex and that $K_{0}=K$.

Extensions of the concept of floating body to spherical and hyperbolic space can be found in $[6,7]$.

Both, the surface body and the weighted floating body, which we introduce next, are variants of the floating body. The surface body and the weighted floating body are relevant in approximation of convex bodies by polytopes.

The surface body was introduced in [36].

Definition 5. [36] Let $s \in \mathbb{R}, s>0$, and let $f: \partial K \rightarrow \mathbb{R}$ be a nonnegative, integrable function with $\int_{\partial K} f d \mu=1$.

The surface body $K_{f, s}$ is the intersection of all the closed half-spaces $\mathrm{H}^{+}$ whose defining hyperplanes $H$ cut off a set of $\mathbb{P}_{f}$-measure less than or equal to $s$ from $\partial K$. More precisely,

$$
K_{f, s}=\bigcap_{\mathbb{P}_{f}\left(\partial K \cap H^{-}\right) \leq s} H^{+}
$$

It follows from the Hahn-Banach theorem that $K_{f, 0} \subseteq K$. If in addition $f$ is almost everywhere nonzero, then $K_{f, 0}=K$.

Another variant of the floating body is the weighted floating body, introduced in [37]. In the following definition $m$ is the Lebesgue measure on $\mathbb{R}^{n}$.

Definition 6. [37] Let $s>0$ and let $f: K \rightarrow \mathbb{R}$ be a nonnegative, integrable function with $\int_{K} f d m=1$, where $m$ is the Lebesgue measure on $\mathbb{R}^{n}$.

The weighted floating body $F(K, f, s)$ is the intersection of all the closed halfspaces $H^{+}$whose defining hyperplanes $H$ cut off a set of $(f \cdot m)$-measure less than or equal to s from $K$. More precisely,

$$
F(K, f, s)=\bigcap_{\int_{K \cap H^{-}} f d m \leq s} H^{+} .
$$

\subsection{Surface body and $L_{p}$ affine surface area}

As noted, a priori the $L_{p}$ affine surface areas are only defined for sufficiently smooth bodies. It was shown in [34] that for any convex body $K$ in $\mathbb{R}^{n}$,

$$
\int_{\partial K} \kappa(x)^{\frac{1}{n+1}} d \mu_{K}(x)=2\left(\frac{\operatorname{vol}_{n-1}\left(B_{2}^{n-1}\right)}{n+1}\right)^{\frac{2}{n+1}} \lim _{\delta \rightarrow 0} \frac{\operatorname{vol}_{n}(K)-\operatorname{vol}_{n}\left(K_{\delta}\right)}{\delta^{\frac{2}{n+1}}},
$$

where $\kappa$ is the generalized Gauss curvature, see e.g., [35]. Thus the right hand side of this equation can be used as a definition of affine surface area which is now valid for all convex bodies. 
Using the surface body or the weighted floating body, extensions of $L_{p}$ affine surface area to general convex bodies can be achieved. The crucial step to do this is the next theorem, for which we need the notion of rolling function $r$ which was introduced in [34] as

$$
r: \partial K \rightarrow \mathbb{R}_{+}, \quad r(x)=\sup \left\{\rho \mid B_{2}^{n}(x-\rho N(x), \rho) \subseteq K\right\},
$$

if $K$ has a unique normal at $x$. If $K$ does not have a unique normal at $x$ then $r(x)=0$.

The rolling function allows to give a quantitive version of Blaschke's rolling theorem which states that for every convex body $K$ with $C^{2}$ boundary and everywhere strictly positive and bounded Gaussian curvature, there is a Euclidean ball with sufficiently small radius $r$ that can roll freely inside the convex body $K$. The latter means that for any point $x \in \partial K$ there is $y \in K$ such that

$$
x \in B_{2}^{n}(y, r) \quad \text { and } \quad B_{2}^{n}(y, r) \subseteq K .
$$

The quantitative version of this theorem is next.

Theorem 7. [34] Let $K$ be a convex body in $\mathbb{R}^{n}$ such that it contains $B_{2}^{n}$. Then we have for all $t$ with $0 \leq t \leq 1$, that $\{x \in \partial K \mid r(x) \geq t\}$ is a closed set and

$$
(1-t)^{n-1} \operatorname{vol}_{n-1}(\partial K) \leq \operatorname{vol}_{n-1}(\{x \in \partial K \mid r(x) \geq t\}) .
$$

The inequality is optimal.

In particular, the function $r^{-\alpha}: \partial K \rightarrow \mathbb{R}$ is Lebesgue integrable for all $\alpha$ with $0 \leq \alpha<1$.

Another notion that is needed is that of minimal function, which was introduced in [36]. Let $f: \partial K \rightarrow \mathbb{R}$ be an integrable, almost everywhere strictly positive function. For $x \in \partial K$ and $s>0$ we put $x_{s}=[0, x] \cap \partial K_{f, s}$. Then the function $M_{f}: \partial K \rightarrow \mathbb{R}$

$$
M_{f}\left(x_{0}\right)=\inf _{0<s} \frac{1}{\operatorname{vol}_{n-1}\left(\partial K \cap H^{-}\left(x_{s}, N_{K_{s}}\left(x_{s}\right)\right)\right)} \int_{\partial K \cap H^{-}\left(x_{s}, N_{K_{s}}\left(x_{s}\right)\right)} f d \mu_{K}
$$

is the minimal function. Here, $H\left(x_{s}, N_{K_{s}}\left(x_{s}\right)\right)$ is the hyperplane through $x_{s}$ with normal $\left.N_{K_{s}}\left(x_{s}\right)\right)$.

Theorem 8. [36] Let $K$ be a convex body in $\mathbb{R}^{n}$. Suppose that $f: \partial K \rightarrow \mathbb{R}$ is an integrable, almost everywhere strictly positive function such that $\int f d \mu=1$. Assume that

$$
\int_{\partial K} \frac{d \mu_{K}(x)}{\left(M_{f}(x)\right)^{\frac{2}{n-1}} r(x)}<\infty .
$$

Then

$$
d_{n} \lim _{s \rightarrow 0} \frac{\operatorname{vol}_{n}(K)-\operatorname{vol}_{n}\left(K_{f, s}\right)}{s^{\frac{2}{n-1}}}=\int_{\partial K} \frac{\kappa^{\frac{1}{n-1}}}{f^{\frac{2}{n-1}}} d \mu_{\partial K},
$$

where $d_{n}=2\left(\operatorname{vol}_{n-1}\left(B_{2}^{n-1}\right)\right)^{\frac{2}{n-1}}$. 
The next corollary shows that the surface body can be used to extend the definition of $L_{p}$ affine surface area to all convex bodies. To do so, we define for $q,-\infty \leq q \leq \infty, q \neq-n$ the functions $f_{q}: \partial K \rightarrow \mathbb{R}$ as follows. For $q= \pm \infty$, put

$$
f_{ \pm \infty}(x)=\frac{\kappa(x)}{a s_{ \pm \infty}(K)\langle x, N(x)\rangle^{n}}
$$

and for all other values of $q$

$$
f_{q}(x)=\frac{\kappa(x)^{\frac{q}{n+q}}}{a s_{q}(K)\langle x, N(x)\rangle^{\frac{n(q-1)}{n+q}}} .
$$

Corollary 9. [36] Let $K$ be a convex body in $\mathbb{R}^{n}$ with the origin in its interior. Let $-\infty \leq p \leq \infty, p \neq-n$. For $p \neq-1$ let $q=\frac{n-p(n-2)}{p+1}$ and for $p=-1$ let $q=\infty$. Let $f_{q}$ be as in (17) and (18) and assume that it is almost everywhere strictly positive. Assume that

$$
\int_{\partial K} \frac{d \mu_{K}(x)}{\left(M_{f_{q}}(x)\right)^{\frac{2}{n-1}} r(x)}<\infty .
$$

Then

$$
d_{n} \lim _{s \rightarrow 0} \frac{\operatorname{vol}_{n}(K)-\operatorname{vol}_{n}\left(K_{f_{q}, s}\right)}{\left(s a s_{q}(K)\right)^{\frac{2}{n-1}}}=a s_{p}(K) .
$$

Weighted floating bodies can similarly be used to provide geometric interpretations of $L_{p}$ affine surface area. We only quote

Theorem 10. [37] Let $K$ be a convex body in $\mathbb{R}^{n}$. Let $f: K \rightarrow \mathbb{R}$ be a continuous function such that $f \geq c$ on $K$, where $c>0$ is an absolute constant. Then

$$
\alpha_{n} \lim _{s \rightarrow 0} \frac{\operatorname{vol}_{n}(K)-\operatorname{vol}_{n}(F(K, f, s))}{s^{\frac{2}{n+1}}}=\int_{\partial K} \frac{\kappa^{\frac{1}{n-1}}}{f^{\frac{2}{n-1}}} d \mu_{\partial K},
$$

where $\alpha_{n}=2\left(\frac{\operatorname{vol}_{n-1}\left(B_{2}^{n-1}\right)}{n+1}\right)^{\frac{2}{n+1}}$.

\section{Random polytopes of points chosen from a convex body}

\subsection{The main theorem}

Ideally for applications one seeks an algorithm that produces, for a given convex body, a best approximating (in a given metric) polytope. The works by e.g., Gordon, Meyer and Reisner [11], by Lopez and Reisner [17] and by Schütt [33] provide constructions of such algorithms. We want to note that the algorithm in [33] is based on the floating bodies of Section 3. The typical questions in this context are: (i) What is the order of magnitude of the best approximation of a 
convex body $K$ in $\mathbb{R}^{n}$ by a polytope $P$ with a fixed number of vertices?

We will concentrate on this aspect. Moreover, here we will only consider approximating polytopes that are inscribed in the body $K$. Arbitrary positioned approximating polytopes were considered in $[14,20]$. If one considers such a setup, then one gains by a factor of dimension. (ii) What is the order of magnitude

of the best approximation of a convex body $K$ in $\mathbb{R}^{n}$ by a polytope $P$ with a fixed number of $(n-1)$-dimensional faces or - more generally - by a fixed number of $k$-dimensional faces, $1 \leq k \leq n-1$ ?

For results on that item we refer to the literature. Of course we have to specify

which metric we use in these approximation questions. Here, we only consider the symmetric difference metric $d_{s}$, which, for two convex bodies $K$ and $L$ in $\mathbb{R}^{n}$ is given by

$$
d_{s}=\operatorname{vol}_{n}(K \backslash L \cup L \backslash K) .
$$

There are many other metrics that have been considered. Again we refer to the literature for more on that topic.

Many of the results deal with asymptotic estimates. A typical example is given by the result (1) by Gruber [13] and McClure and Vitale [24] mentioned in the introduction.

But it is only in rare special cases that a best approximating polytope can be explicitly singled out. Consequently, a common practice is to randomize. Consider the random polytope $P_{N}$ obtained as the convex hull of $N$ points chosen at random in the convex body with respect to a probability measure $\mathbb{P}$. A natural choice for the probability measure $\mathbb{P}$ is the normalized Lebesgue measure on $K$. Denote the expected volume of a random polytope of $N$ randomly chosen points by $\mathbb{E}(K, N)$.

A striking example of an asymptotic result of a probabilistic nature is due to Bárány [2] (in case the boundary is $C^{3}$ ), and Schütt [32] in the general case:

$$
c \lim _{N \rightarrow \infty} \frac{\operatorname{vol}_{n}(K)-\mathbb{E}(K, N)}{\left(\frac{\operatorname{vol}_{n}(K)}{N}\right)^{\frac{2}{n+1}}}=\int_{\partial K} \kappa(x)^{\frac{1}{n+1}} d \mu_{K}(x),
$$

where $c>0$ is an absolute constant. Note that the order of magnitude $N^{-2 /(n+1)}$ of the random result is not as good the best order of approximation provided by (1). But this is to be expected: not all points chosen inside $K$ are necessarily vertices of the approximating random polytope.

A more economical way is to choose the points at random with respect to a probability measure directly on the boundary of the body.

The setting is now as follows. For an integrable, nonnegative function $f$ : $\partial K \rightarrow \mathbb{R}$ with $\int_{\partial K} f(x) d \mu_{K}=1$ we denote by $\mathbb{P}_{f}$ the probability measure with $d \mathbb{P}_{f}=f d \mu_{K}$. We considerer random polytopes $P_{N}$ where the points are chosen from the boundary of $K$ with respect to $\mathbb{P}_{f}$. Then the expected volume of such a random polytope is

$$
\mathbb{E}(f, N)=\mathbb{E}\left(\mathbb{P}_{f}, N\right)=\int_{\partial K} \ldots \int_{\partial K} \operatorname{vol}_{n}\left(\left[x_{1}, \ldots, x_{N}\right]\right) d \mathbb{P}_{f}\left(x_{1}\right) \ldots d \mathbb{P}_{f}\left(x_{N}\right),
$$


where $\left[x_{1}, \ldots, x_{N}\right]$ is the convex hull of the points $x_{1}, \ldots, x_{N}$. For such a setting the following theorem was proved in [35]. A crucial ingredient in the proof of this theorem were the surface bodies. This theorem was also proved in [28] under stronger smoothness assumptions on the boundary of $K$.

Theorem 11. [35] Let $K$ be a convex body in $\mathbb{R}^{n}$ such that there are $r$ and $R$ in $\mathbb{R}$ with $0<r \leq R<\infty$ so that we have for all $x \in \partial K$

$$
B_{2}^{n}\left(x-r N_{\partial K}(x), r\right) \subseteq K \subseteq B_{2}^{n}\left(x-R N_{\partial K}(x), R\right)
$$

and let $f: \partial K \rightarrow \mathbb{R}_{+}$be a continuous, positive function with $\int_{\partial K} f(x) d \mu_{\partial K}(x)=$ 1. Let $\mathbb{P}_{f}$ be the probability measure on $\partial K$ given by $\mathbb{P}_{f}(x)=f(x) \mu_{\partial K}(x)$. Then we have

$$
\lim _{N \rightarrow \infty} \frac{\operatorname{vol}_{n}(K)-\mathbb{E}(f, N)}{\left(\frac{1}{N}\right)^{\frac{2}{n-1}}}=c_{n} \int_{\partial K} \frac{\kappa(x)^{\frac{1}{n-1}}}{f(x)^{\frac{2}{n-1}}} d \mu_{\partial K}(x)
$$

where $\kappa$ is the (generalized) Gauß-Kronecker curvature and

$$
c_{n}=\frac{(n-1)^{\frac{n+1}{n-1}} \Gamma\left(n+1+\frac{2}{n-1}\right)}{2(n+1) !\left(\operatorname{vol}_{n-2}\left(\partial B_{2}^{n-1}\right)\right)^{\frac{2}{n-1}}} .
$$

The minimum at the right hand side is attained for the normalized affine surface area measure with density

$$
f_{a s}(x)=\frac{\kappa(x)^{\frac{1}{n+1}}}{\int_{\partial K} \kappa(x)^{\frac{1}{n+1}} d \mu_{\partial K}(x)} .
$$

As the random polytope $P_{N} \subseteq K$, all the above results are approximation results in the symmetric difference metric. The condition: there are $r$ and $R$ in $\mathbb{R}$ with $0<r \leq R<\infty$ so that we have for all $x \in \partial K$

$$
B_{2}^{n}\left(x-r N_{\partial K}(x), r\right) \subseteq K \subseteq B_{2}^{n}\left(x-R N_{\partial K}(x), R\right)
$$

is satisfied if $K$ has a $C^{2}$-boundary with everywhere positive curvature. This follows from Blaschke's rolling theorem [5], respectively it's generalization, Theorem 7 , of [34]. Indeed, we can choose

$$
r=\min _{x \in \partial K} \min _{1 \leq i \leq n-1} r_{i}(x) \quad R=\max _{x \in \partial K} \max _{1 \leq i \leq n-1} r_{i}(x),
$$

where $r_{i}(x)$ denotes the $i$-th principal curvature radius. By a result of Alexandroff [1], the generalized curvature $\kappa$ exists a.e. on a convex body. It was shown in [34] that $\kappa^{\frac{1}{n+1}}$ is an integrable function. Therefore the density

$$
f_{a s}(x)=\frac{\kappa(x)^{\frac{1}{n+1}}}{\int_{\partial K} \kappa(x)^{\frac{1}{n+1}} d \mu_{\partial K}(x)}
$$


exists provided that $\int_{\partial K} \kappa(x)^{\frac{1}{n+1}} d \mu_{K}(x)>0$. This is certainly assured by the assumption (20) on the boundary of $K$.

Hence, by this theorem, we get the best random approximation if we choose the points on the boundary of $K$ with respect to the affine surface area measure $\mathbb{P}_{f_{a s}}$. Then the order of magnitude for this random approximation is

$$
c_{n}\left(\int_{\partial K} \kappa(x)^{\frac{1}{n+1}} d \mu(x)\right)^{\frac{n+1}{n-1}} N^{-2 /(n-1)} .
$$

It is natural to see how this best random approximation compares with best approximation.

\subsection{An analysis of the main theorem}

\subsubsection{Comparison: Best approximation and random approximation}

Recall that by (1) best approximation is of the order

$$
\frac{1}{2} \operatorname{del}_{n-1}\left(\int_{\partial K} \kappa(x)^{\frac{1}{n+1}} d \mu_{K}(x)\right)^{\frac{n+1}{n-1}} N^{-2 /(n-1)} .
$$

Thus, to see how random approximation (22) compares to the best approximation (23), we only need to compare $c_{n}$ and $\frac{1}{2} \operatorname{del}_{n-1} . c_{n}$ is given by (21). $\operatorname{del}_{n-1}$ was determined in a series of papers by Gordon, Reisner and Schütt [12] and Mankiewicz and Schütt [22,23]. Using these results, one gets that with an absolute constant $c>0$,

$$
\frac{1}{2} \operatorname{del}_{n-1} \leq c_{n} \leq\left(1+c \frac{\ln n}{n}\right) \frac{1}{2} \operatorname{del}_{n-1} .
$$

Therefore, surprisingly, random approximation is (almost) as good as best approximation.

\subsubsection{Other measures $\mathbb{P}_{f}$}

Aside from the best approximating measure $\mathbb{P}_{f_{a s}}$, there are other measures of interest. We list some of them.

1. The second measure of interest is the surface measure given by the constant density

$$
f_{s}(x)=\frac{1}{\operatorname{vol}_{n-1}(\partial K)} .
$$

This measure is not affine invariant and we get

$$
\lim _{N \rightarrow \infty} \frac{\operatorname{vol}_{n}(K)-\mathbb{E}\left(f_{s}, N\right)}{\left(\frac{\operatorname{vol}_{n-1}(\partial K)}{N}\right)^{\frac{2}{n-1}}}=c_{n} \int_{\partial K} \kappa(x)^{\frac{1}{n-1}} d \mu_{K}(x) .
$$


2. The next measure is the normalized cone measure, see also (10). Recall that we assume throughout without loss of generality that 0 is the center of gravity of a convex body $K$. Let $A$ be a subset of $\partial K$ and denote by $[0, A]$ the convex hull of 0 and $A$. Then the normalized cone measure is given by

$$
\mathbb{P}(A)=\frac{\operatorname{vol}_{n}([0, A])}{\operatorname{vol}_{n}(K)},
$$

As $\frac{1}{n} \int_{\partial K}\langle x, N(x)\rangle d \mu_{K}(x)=\operatorname{vol}_{n}(K)$, its density is

$$
f_{c}(x)=\frac{\langle x, N(x)\rangle}{\int_{\partial K}\langle x, N(x)\rangle d \mu_{K}(x)}=\frac{\langle x, N(x)\rangle}{n \operatorname{vol}_{n}(K)}=q_{k}(x),
$$

where $q_{k}$ is as in (10). The measure is invariant under linear, volume preserving maps. We get

$$
\lim _{N \rightarrow \infty} \frac{\operatorname{vol}_{n}(K)-\mathbb{E}\left(f_{c}, N\right)}{\left(\frac{n \operatorname{vol}_{n}(K)}{N}\right)^{\frac{2}{n-1}}}=c_{n} \int_{\partial K} \frac{\kappa(x)^{\frac{1}{n-1}}}{\langle x, N(x)\rangle^{\frac{2}{n-1}}} d \mu_{K}(x) .
$$

Recalling the $L_{p}$ affine surface area of Section 2, we observe that the expression on the right hand side of (24) is exactly $a s_{p=n /(n-2)}$.

Thus one is naturally led to suppose that other $L_{p}$ affine surface areas might appear in the setting of Theorem 11.

\subsubsection{Random polytopes and $L_{p}$ affine surface areas}

We want to present a geometric characterization of the $L_{p}$ affine surface area for all $p$ similar in spirit to the one given in Corollary 9 .

For $q,-\infty \leq q \leq \infty, q \neq-n$, let the functions $f_{q}: \partial K \rightarrow \mathbb{R}$ be given as in (17) and (18). Then the following corollary is an immediate consequence of Theorem 11.

Corollary 12. Let $K$ be a convex body in $\mathbb{R}^{n}$ with the origin in its interior. Assume also that there are $r$ and $R$ in $\mathbb{R}$ with $0<r \leq R<\infty$ so that we have for all $x \in \partial K$

$$
\begin{gathered}
B_{2}^{n}(x-r N(x), r) \subseteq K \subseteq B_{2}^{n}(x-R N(x), R) . \\
\text { Let }-\infty \leq p \leq \infty, p \neq-n \text {. For } p \neq-1 \text { let } q=\frac{n-p(n-2)}{p+1} . \text { Then } \\
\lim _{N \rightarrow \infty} \frac{\operatorname{vol}_{n}(K)-\mathbb{E}\left(f_{q}, N\right)}{\left(\frac{a s_{q}(K)}{N}\right)^{\frac{2}{n-1}}}=c_{n} a s_{p}(K) .
\end{gathered}
$$

And

$$
\lim _{N \rightarrow \infty} \frac{\operatorname{vol}_{n}(K)-\mathbb{E}\left(f_{ \pm \infty}, N\right)}{\left(\frac{a s_{ \pm \infty}(K)}{N}\right)^{\frac{2}{n-1}}}=c_{n} a s_{-1}(K)
$$

where $c_{n}=\frac{(n-1)^{\frac{n+1}{n-1}} \Gamma\left(n+1+\frac{2}{n-1}\right)}{2(n+1) !\left(\operatorname{vol}_{n-2}\left(\partial B_{2}^{n-1}\right)\right)^{\frac{2}{n-1}}}$. 
Thus Corollary 12 can be viewed as a geometric interpretation, in the spirit of Corollary 9 , of $L_{p}$ affine surface areas via random polytopes.

\section{Acknowledgments}

The author wants to thank the referees for the careful reading and suggestions for improvement.

\section{References}

[1] A.D. Alexandroff, Almost everywhere existence of the second differential of a convex function and some properties of convex surfaces connected with it, (Russian) Leningrad State Univ. Annals [Uchenye Zapiski] Math. Ser. 6 (1939) 3-35. MR0003051

[2] I. BÁrÁny, Random polytopes in smooth convex bodies. Mathematika 39 (1992) 81-92 MR1176473

[3] I. BÁrÁny And D.G. LARman, Convex bodies, economic cap coverings, random polytopes, Mathematika 35 (1988) 274-291 MR0986636

[4] A. Barvinok And E. VeOMetT, The computational complexity of convex bodies, Surveys on Discrete and Computational Geometry, Contemporary Mathematics, 453 (2008) 17-137 MR2405680

[5] W. Blaschke, Vorlesungen über Differentialgeometrie II: Affine Differentialgeometrie, Springer Verlag, Berlin, (1923) MR1548296

[6] F. BesAu AND E.M. WERnER, The spherical convex floating body, Adv. Math. 301 (2016) 867-901 MR3539392

[7] F. Besau And E.M. Werner, The floating body in real space forms, Journal Diff. Geometry, 110 (2018) 187-220 MR3861810

[8] T. Cover And J. Thomas, Elements of information theory, second ed., Wiley-Interscience, (John Wiley and Sons), Hoboken, NJ, (2006). MR2239987

[9] H. Edelsbrunner, Geometric algorithms. Handbook of Convex Geometry, 699-735. North Holland, (1993) MR1242994

[10] R. J. Gardner, Geometric Tomography. Cambridge University Press, (1995) MR1356221

[11] Y. Gordon, M. Meyer And S. ReISner, Constructing a polytope to approximate a convex body Geometriae Dedicata 57 (1995) 217-222 MR1347327

[12] Y. Gordon, S. Reisner And C. SchütT, Umbrellas and polytopal approximation of the Euclidean ball, Journal of Approximation Theory 90 (1997) 9-22 MR1458818

[13] P. M. Gruber Approximation of convex bodies, In Convexity and its Applications, 131-162 Birkhäuser, Basel, 1983. MR0731110

[14] J. Grote And E.M. Werner, Approximation of smooth convex bodies by random polytopes, Journal of Probability 23 (2018) 1-21 MR3771746 
[15] Handbook on the geometry of Banach spaces, Vol 1, 2, W.B. Johnson, J. Lindenstrauss eds. Elsevier Science, (2003) MR1863689

[16] F. Liese AND I. VAJdA, On Divergences and Information in Statistics and Information Theory, IEEE Trans. Inform. Theory 52 (2006) 4394 4412 MR2300826

[17] M. A. Lopez AND S. ReISNER, Efficient approximation of convex polygons, International Journal of Computational Geometry and Applications 10 (2000) 445-452 MR1793666

[18] L. LovÁsz AND M. Simonovits, Random walks in a convex body and an improved volume algorithm. Random structures and algorithms 4 (1993) 359-412 MR1238906

[19] L. LovÁsz AND S. Vempala Simulated annealing in convex bodies and an $O^{*}\left(n^{4}\right)$ volume algorithm. J. Comput. System Sci. 72 (2006) 392-417 MR2205290

[20] M. Ludwig, C. Schütt And E. M. Werner, Approximation of the Euclidean ball by a polytope, Studia Mathematica 173 (2006) 1-18 MR2204459

[21] E. Lutwak, The Brunn-Minkowski-Firey theory. II. Affine and geominimal surface areas, Adv. Math. 118 (1996) 244-294 MR1378681

[22] P. Mankiewicz And C. SchÜtT, A simple proof of an estimate for the approximation of the Euclidean ball and the Delone triangulation numbers. Journal of Approximation Theory, 107, 268-280 MR1806953

[23] P. Mankiewicz and C. Schütt, On the Delone triangulations numbers, Journal of Approximation Theory 111 (2011) 139-142 MR1840025

[24] D. McClure and R. Vitale Polygonal approximation of plane convex bodies, J. Math. Anal. Appl. 51 (1975) 326-358 MR0385714

[25] M. MEYer AND E.M. Werner, On the p-affine surface area Adv. Math. 152 (2000) 288-313 MR1764106

[26] G. PAOURIS AND E. M. Werner On the approximation of a polytope by its dual $L_{p}$-centroid bodies, Indiana Univ. Math. J. 62 (2013) 235-247 MR3158508

[27] J. Prochno, C. Schütt And E. M. Werner, Best and random approximation of a convex body by a polytope, J. of Complexity doi.org/10.1016/j.jco.2022.101652

[28] M. Reitzner, Random points on the boundary of smooth convex bodies, Transactions of the American Mathematical Society 354 (2002) 2243-2278 MR1885651

[29] A. RÉNYI, On measures of entropy and information, Proceedings of the 4th Berkeley Symposium on Probability Theory and Mathematical Statistics 1 (1961) 547-561 MR0132570

[30] G. SaPiro And A. TAnnenbaum, On invariant curve evolution and image analysis. Indiana Univ. Journal of Math. 42 (1994) 985-1009 MR1254129

[31] R. SchneIder, Convex bodies: The Brunn-Minkowski theory, 2nd expanded edition, Cambridge University Press, Cambridge, (2014) MR3155183

[32] C. SснÜтт, The convex floating body and polyhedral approximation, Israel J. Math. 73 (1991) 65-77 MR1119928 
[33] C. SchÜтt, Floating body, illumination body, and polytopal approximation, in: K.M. Ball und V. Milman: Convex Geometric Analysis, Mathematical Sciences Research Institute Publications 34, Cambridge University Press,(1999) 203-230 MR1665593

[34] C. Schütt And E. M. Werner, The convex floating body, Math. Scand. 66 (1990) 275-290 MR1075144

[35] C. SchÜtt And E.M. Werner, Polytopes with vertices chosen randomly from the boundary of a convex body, GAFA Seminar Notes, Lecture Notes in Mathematics 1807, Springer-Verlag (2003) 241-422 MR2083401

[36] C. Schütt And E.M. Werner, Surface bodies and p-affine surface area, Adv. Math. 187 (2004) 98-145 MR2074173

[37] E.M. WERNER, The p-affine surface area and geometric interpretations, Rend. Circ. Mat. Palermo (2) Suppl. (2002), no. 70, part II, 367-382 MR1962608

[38] E.M. WERNER, Rényi divergence and $L_{p}$-affine surface area for convex bodies, Adv. Math., 230 (2012) 1040-1059 MR2921171

[39] E.M. Werner And D. Ye, New $L_{p}$ affine isoperimetric inequalities, Adv. Math. 218 (2008) 762-780 MR2414321 